\title{
Angiographic dimple of profound significance in cases of aneurysmal subarachnoid hemorrhage: report of $\mathbf{2}$ cases
}

\author{
Jaechan Park, MD, PhD \\ Department of Neurosurgery, Kyungpook National University, Daegu, Republic of Korea
}

The occurrence of an angiographic dimple or irregularity due to indentation of the contrast column by an intraluminal thrombus at the dome of a ruptured aneurysm is not uncommon and does not draw much clinical attention. However, an angiographic dimple at the base of the ruptured aneurysm or division of the parent artery can signify a rupture point close to the dimple and an intraluminal thrombus, which has utmost clinical significance as it is close to the parent artery and necessitates a different treatment strategy from rupture of the aneurysm dome. The author reports on 2 cases of an angiographic dimple following subarachnoid hemorrhage (SAH) and subsequent surgical exploration. In the first patient, a 57-year-old-woman, angiography revealed a basal dimple in a superiorly directed anterior communicating artery aneurysm. A pterional craniotomy was performed, which revealed a bilobed aneurysm harboring a superiorly directed unruptured lobule and inferiorly directed ruptured lobule. An intraluminal thrombus in the inferiorly directed lobule apparently obscured the lobule and caused the appearance of the basal dimple on the angiograms. In the second patient, a 40-year-old man who had been transferred to the author's institution because of an angiographic evaluation that did not show any aneurysm despite SAH in the basal cisterns, initial angiography revealed a subtle dimple on the superior wall of the anterior communicating artery (ACoA). On follow-up angiography, a very small aneurysm was seen at the site of the dimple. A craniotomy then revealed a very small ruptured and thrombosed aneurysm on the superior wall of the ACoA.

http://thejns.org/doi/abs/10.3171/2014.11.JNS142319

KEY WORDS aneurysm; angiogram; subarachnoid hemorrhage; thrombus; vascular disorders

A RUPTURED cerebral aneurysm can achieve hemostasis when a thrombus develops and seals the rupture point of the aneurysm. If the thrombus occupies the lumen of the aneurysm, the angiographic configuration is distorted due to indentation of the contrast column. An angiographic dimple in a ruptured aneurysm can also appear due to an intraluminal thrombus.

Since most aneurysm ruptures occur in the wall of the aneurysm dome, an angiographic dimple or irregularity of the dome is not uncommon and does not draw much clinical attention. However, an angiographic dimple at the base of the ruptured aneurysm or a division of the parent artery can imply a rupture point close to the parent artery and an intraluminal thrombus. This has utmost clinical significance, as a location close to the parent artery requires a treatment strategy different from that of rupture of the aneurysm dome. Here, 2 cases of an angiographic dimple with profound significance in subsequent surgical exploration are reported.

\section{Case Reports}

\section{Case 1: Ruptured ACoA Aneurysm With a Dimple in the Aneurysm Base}

Examination

A 57-year-old woman presented with a sudden bursting headache; CT scanning showed an subarachnoid hemorrhage (SAH) in the basal cisterns. Subsequent CT angiography (CTA) and digital subtraction angiography (DSA) revealed a saccular anterior communicating artery (ACoA) aneurysm with a maximum diameter of $7 \mathrm{~mm}$, arising at the left $\mathrm{A}_{1}-\mathrm{A}_{2}$ junction and directed superiorly (Fig. 1A and B). Because of the angiographic presence of a dimple at the base of the aneurysm, surgical treatment was favored over endovascular coiling due to the possibility of a basal rupture.

\section{Operation}

A left pterional craniotomy revealed an ACoA aneu-

ABBREVIATIONS ACOA = anterior communicating artery; CTA = CT angiography; DSA = digital subtraction angiography; $\mathrm{SAH}=$ subarachnoid hemorrhage. SUBMITTED October 9, 2014. ACCEPTED November 11, 2014.

INCLUDE WHEN CITING Published online May 22, 2015; DOI: 10.3171/2014.11.JNS142319.

DISCLOSURE The author reports no conflict of interest concerning the materials or methods used in this study or the findings specified in this paper. 

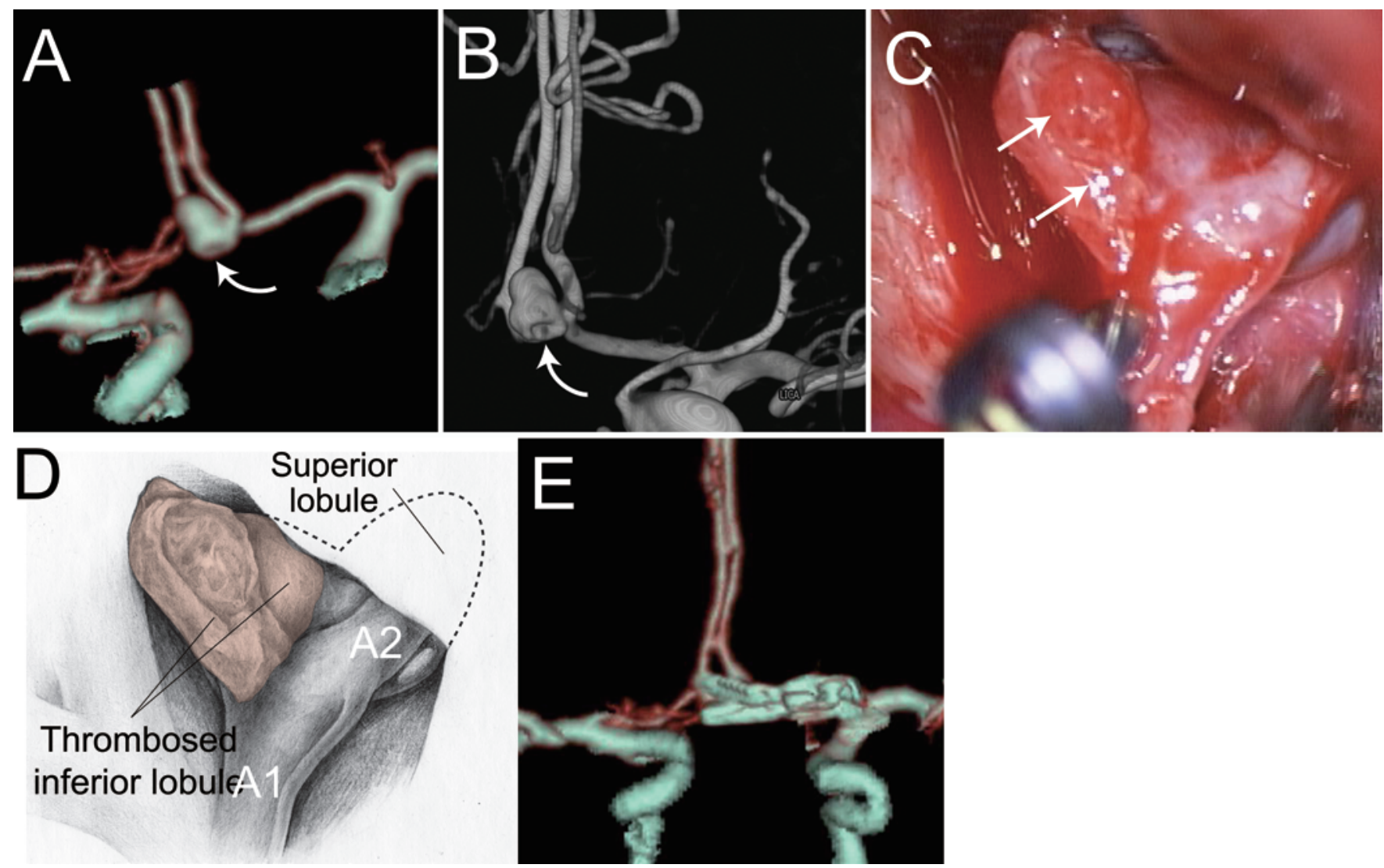

FIG. 1. Case 1. A: CT angiogram. The arrow indicates the dimple of the aneurysm. B: DS angiogram showing a superiorly directed saccular aneurysm at the left $A_{1}-A_{2}$ junction. Note the dimple (arrow) at the base of the aneurysm. C: Intraoperative photograph showing a bilobed aneurysm with an unruptured superiorly directed lobule between the bilateral $A_{2}$ segments and ruptured, thrombosed inferiorly directed lobule. Note the rupture site of the inferiorly directed lobule (arrows). D: Corresponding illustration. Copyright Jaechan Park. Published with permission. E: Postoperative CT angiogram obtained after clipping of both lobules separately.

rysm with a bilobed shape, which differed from the configuration seen on angiography. The superiorly directed lobule corresponded to the ACoA aneurysm seen on the angiograms and was noted to be unruptured, while the inferiorly directed lobule included a large rupture area involving the aneurysmal body and base and was thrombosed (Fig. 1C and 1D). An intraluminal thrombus in the inferiorly directed lobule had apparently obscured this lobule and produced the basal dimple seen on angiography. Both lobules were clipped separately.

\section{Postoperative Course}

The patient awoke from the surgery without neurological deficits and returned to normal life. The postoperative and follow-up CT angiograms showed no evidence of a residual aneurysm (Fig. 1E).

\section{Case 2: Subtle Dimple on the Superior Wall of the ACoA \\ Examination}

A 40-year-old man was transferred from another hospital. At that institution he had undergone angiographic evaluation for $\mathrm{SAH}$, but no aneurysm was visualized on the angiogram. The patient presented with a sudden bursting headache, and the CT scan showed a diffuse SAH in the basal cisterns, which was predominant in the anterior interhemispheric fissure, while DSA revealed no intracranial aneurysms or dissections. However, due to a subtle angiographic dimple on the superior wall of the ACoA (Fig. 2A), early follow-up DSA was scheduled on the 5th day after $\mathrm{SAH}$, which revealed a very small saccular aneurysm at the site of the subtle dimple on the superior wall of the ACoA (Fig. 2B and 2C).

\section{Operation}

A left pterional craniotomy revealed a small reddish thrombus attached to the superior wall of the ACoA (Fig. 2D). Removal of the thrombus revealed a tiny opening with a diameter less than $1 \mathrm{~mm}$ on the superior wall of the ACoA. This vascular opening was closed by applying an aneurysm clip across the ACoA.

\section{Postoperative Course}

The postoperative recovery was uneventful, and the patient returned to normal life. A control DS angiogram and follow-up CT angiogram obtained at 6 months showed successful obliteration of the aneurysm (Fig. 2E). The blood flow into the distal part of the bilateral anterior cerebral arteries was normally preserved through the ipsilateral $\mathrm{A}_{1}$ segments. 

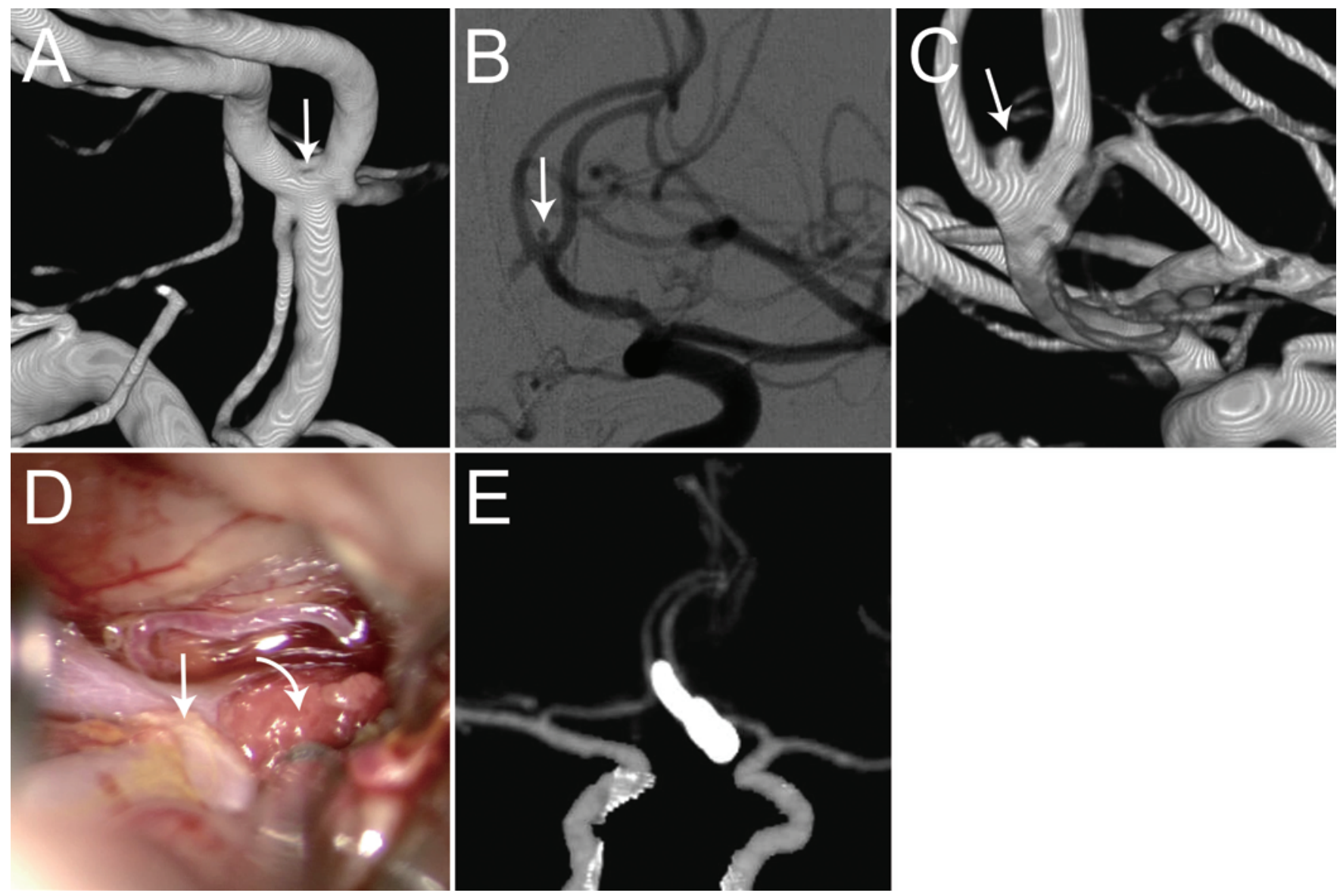

FIG. 2. Case 2. A: Initial DS angiogram showing a subtle dimple (arrow) on the superior wall of the ACoA. B and C: DS angiograms revealing a very small aneurysm (arrow) at the site of the dimple on the superior wall of the ACoA. D: Intraoperative photograph showing a small reddish thrombus (curved arrow) attached to the superior wall of the ACOA (straight arrow). E: Postoperative CT angiogram.

\section{Discussion}

While most cerebral aneurysms rupture in the dome, the occasional occurrence of basal rupture is also well known. Literature on basal ruptures, however, is scarce. ${ }^{1,3,4,6,8,9}$ Crompton's study describing autopsies found that only about $2 \%$ of ruptures occurred in the basal area of the aneurysm. ${ }^{1}$ Meanwhile, my colleagues and I previously reported the appearance of a small basal outpouching on approximately $9 \%$ of angiograms, the most common angiographic sign suggesting the possibility of an aneurysmal basal rupture, where one-third of this surgical subseries was subsequently found to have a basal rupture. ${ }^{9}$

Ruptured aneurysms commonly achieve hemostasis mainly via an extraaneurysmal thrombus surrounding the rupture point. However, an intraaneurymsal thrombus can be a major mechanism for sealing a rupture point. Ishikawa et al. ${ }^{5}$ reported a $10 \%$ surgical incidence of hemostasis of a ruptured aneurysm that included an inside-arrest pattern with a thrombus attached to the rupture point from inside the aneurysm. If an intraaneurysmal thrombus seals a basal rupture, it indents the contrast column on angiography, creating a peculiar configuration that can include a stalk-like narrow neck or basal dimple. ${ }^{8}$

A basal rupture of an aneurysm should be treated sur- gically rather than with endovascular coiling. According to previous reports, ${ }^{6,9}$ cases of a ruptured aneurysm with a small basal outpouching had a high incidence of intraprocedural rupture during coil embolization. In addition, an intraaneurysmal thrombus sealing a basal rupture prevents sufficient coil packing of the aneurysm between the rupture point and the parent artery, increasing the risk of recurrent hemorrhage after coiling. In contrast, surgical treatment can achieve optimal results when appropriate techniques are used, such as temporary aneurysm trapping instead of proximal artery clipping, cautious neck dissection after dissecting the other parts of the aneurysm, and clip placement that includes the wall of the parent artery or aneurysm neck clipping following microvascular suture repair of the injured parent artery.

However, an intraluminal thrombus is not the only cause of an angiographic dimple. A posterior communicating artery infundibulum can exhibit a dimpled appearance on DSA in the central aspect of its dome during the early phase of internal carotid artery injection due to the presence of unopacified blood within the infundibulum from the posterior communicating artery. ${ }^{7}$ Meanwhile, arterial fenestrations of the circle of Willis can appear, ranging from 2 parallel vessels to a subtle dimple. According 
to a study using 3D rotational angiography by de Gast et al., ${ }^{2}$ fenestrations of the ACoA were observed in about $5 \%$ of cases and included a wide variety of configurations. However, since ACoA fenestrations are visible on anteroposterior views of angiograms, the location of a dimple is a crucial differential point between ACoA fenestration and a ruptured ACOA aneurysm.

\section{Conclusions}

An angiographic dimple can have a profound clinical significance in SAH patients. In particular, an angiographic dimple at the aneurysm base or a division of the parent artery can imply a rupture point close to the parent artery and an intraluminal thrombus.

\section{References}

1. Crompton MR: Mechanism of growth and rupture in cerebral berry aneurysms. BMJ 1:1138-1142, 1966

2. de Gast AN, van Rooij WJ, Sluzewski M: Fenestrations of the anterior communicating artery: incidence on 3D angiography and relationship to aneurysms. AJNR Am J Neuroradiol 29:296-298, 2008

3. Fisher CM, Ojemann RG: Basal rupture of saccular aneurysm. A pathological case report. J Neurosurg 48:642-644, 1978
4. Frerichs KU, Stieg PE, Friedlander RM: Prediction of aneurysm rupture site by an angiographically identified bleb at the aneurysm neck. J Neurosurg 93:517, 2000

5. Ishikawa T, Nakayama N, Yoshimoto T, Aoki T, Terasaka S, Nomura M, et al: How does spontaneous hemostasis occur in ruptured cerebral aneurysms? Preliminary investigation on 247 clipping surgeries. Surg Neurol 66:269-276, 2006

6. Kang DH, Goh DH, Baik SK, Park J, Kim YS: Morphological predictors of intraprocedural rupture during coil embolization of ruptured cerebral aneurysms: do small basal outpouchings carry higher risk? J Neurosurg 121:605-612, 2014

7. Kaufmann TJ, Razack N, Cloft HJ, Kallmes DF: Dimpled appearance of a posterior communicating artery saccule: an angiographic indicator of arterial infundibula. AJR Am J Roentgenol 185:1358-1360, 2005

8. Park J: Saccular aneurysm with basal rupture angiographically depicted as an aneurysm with stalk-like narrow neck. J Neurosurg 114:1065-1068, 2011

9. Park J, Woo H, Kang DH, Kim Y, Baik SK: Ruptured intracranial aneurysms with small basal outpouching: incidence of basal rupture and results of surgical and endovascular treatments. Neurosurgery 71:994-1002, 2012

\section{Correspondence}

Jaechan Park, Department of Neurosurgery, Kyungpook National University Hospital, 50, Samduk 2-ga, Jung-gu, Daegu 700-721, South Korea. email: jparkmd@hotmail.com. 